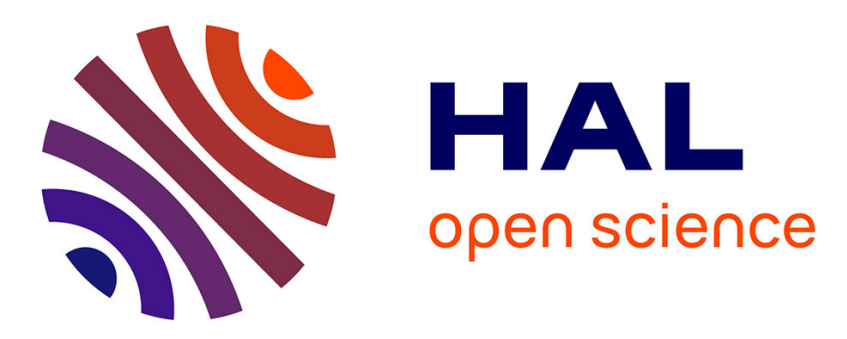

\title{
Mode propagation in curved waveguides and scattering by inhomogeneities: application to the elastodynamics of helical structures
}

Fabien Treyssède

\section{- To cite this version:}

Fabien Treyssède. Mode propagation in curved waveguides and scattering by inhomogeneities: application to the elastodynamics of helical structures. Journal of the Acoustical Society of America, 2011, 129, pp.1857-1868. hal-01066382

\section{HAL Id: hal-01066382 \\ https://hal.science/hal-01066382}

Submitted on 19 Sep 2014

HAL is a multi-disciplinary open access archive for the deposit and dissemination of scientific research documents, whether they are published or not. The documents may come from teaching and research institutions in France or abroad, or from public or private research centers.
L'archive ouverte pluridisciplinaire HAL, est destinée au dépôt et à la diffusion de documents scientifiques de niveau recherche, publiés ou non, émanant des établissements d'enseignement et de recherche français ou étrangers, des laboratoires publics ou privés. 
Mode propagation in curved waveguides and scattering by inhomogeneities: application to the elastodynamics of helical structures

Fabien Treyssède ${ }^{a)}$

IFSTTAR,

Centre de Nantes,

BP4129,

44341 Bouguenais Cedex,

France

(Dated: January 26, 2011)

Mode scattering in curved waveguides 1 


\begin{abstract}
This paper reports on an investigation into the propagation of guided modes in curved waveguides and their scattering by inhomogeneities. In a general framework, the existence of propagation modes traveling in curved waveguides is discussed. The concept of translational invariance, intuitively used for the analysis of straight waveguides, is highlighted for curvilinear coordinate systems. Provided that the crosssection shape and medium properties do not vary along the waveguide axis, it is shown that a sufficient condition for invariance is the independence on the axial coordinate of the metric tensor. Such a condition is indeed checked by helical coordinate systems. This study then focuses on the elastodynamics of helical waveguides. Given the difficulty in achieving analytical solutions, a purely numerical approach is chosen based on the so-called semi-analytical finite element method. This method allows the computation of eigenmodes propagating in infinite waveguides. For the investigation of modal scattering by inhomogeneities, a hybrid finite element method is developed for curved waveguides. The technique consists in applying modal expansions at cross-section boundaries of the finite element model, yielding transparent boundary conditions. The final part of this paper deals with scattering results obtained in free-end helical waveguides. Two validation tests are also performed.
\end{abstract}

PACS numbers: 43.35.Cg, 43.20.Mv, 43.20.Bi 


\section{INTRODUCTION}

Many applications are likely to involve guided wave propagation inside curved structures. However the literature concerning the theory of curved waveguides is rather scarce compared to straight waveguides. For instance, some references can be found on elastic waves propagating in circular plates ${ }^{1-3}$, toroidal pipes ${ }^{4}$, tyres $^{5}$, spherical plates ${ }^{6,7}$, circular waveguides of arbitrary cross-section ${ }^{8}$, twisted bars $^{9}$, helical springs ${ }^{10-12}$, multi-wire helical strands $^{13}$ as well as electromagnetic waves in optical fibers ${ }^{14-16}$.

The goal of this paper is to highlight the propagation of modes in curved waveguides as well as their scattering by inhomogeneities. The existence of propagation modes traveling in infinite curved waveguides is first discussed in a general framework (Sec. II), valid for any type of waves (elastic, acoustic, electromagnetic...).

Then, the remainder of the paper focuses on the elastodynamics of helical waveguides. In the context of non-destructive testing and structural health monitoring of cables ${ }^{17-21}$, one of the motivations is to develop modeling tools for progress in the understanding of wave-damage interaction in helical structures. The basic element of civil-engineering cables is usually a seven-wire strand, consisting of one straight cylindrical core wire surrounded by one layer of six helical wires. The results of this paper will only concern single wires, inter-wire contact effects being left for further investigations.

Given the difficulty in achieving analytical solutions, a purely numerical approach is chosen. The formulation is fully three-dimensional. For the computation of guided modes, the so-called semi-analytical finite element (SAFE) technique is employed. This technique has been extensively used in elasticity for uniform straight waveguides - see for instance Refs. [22-27]. It has also been proposed in duct acoustics ${ }^{28,29}$ and electromagnetics ${ }^{15,30}$. The advantage of SAFE methods is their capacity to handle complex waveguides of arbitrary cross-section, together with material anisotropy and transverse heterogeneity. An axial dependence $e^{i k s}$ is assumed before finite element $(\mathrm{FE})$ discretization $(k$ and $s$ are

\footnotetext{
a) Electronic address: fabien.treyssede@ifsttar.fr
} 
the wavenumber and distance along the waveguide axis, respectively) - hence reducing the problem from three to two dimensions - so that only the cross-section needs to be meshed. Recently, the author has proposed a SAFE method for helical elastic waveguides ${ }^{13,31}$. This method is briefly recalled in this paper (Sec. III) and some dispersion curves are shown for helical waveguides of circular cross-sections with different helix lay angles.

For modal scattering by inhomogeneities, the present paper proposes a hybrid FE-based method (Sec. IV). It consists in applying modal expansions at cross-section boundaries of a usual FE model, where semi-infinite waveguides are connected, yielding transparent boundary conditions. The FE model can then be limited to a small region surrounding the inhomogeneity, which in turn might be of complex shape (cracks, discontinuities, local bends,...). The modal expansions requires the knowledge of guided modes, which can be obtained from analytical solutions when available, or from the SAFE method as done in this paper. The solution of the hybrid system directly yields the coefficients of scattered modes. In the literature, hybrid methods have already been proposed for elastic guided waves in two-dimensional straight waveguides (plates ${ }^{32-37}$ and cylinders ${ }^{38}$ ) as well as acoustic waves in ducts $^{39,40}$ for instance. A close relationship between hybrid and Dirichlet-to-Neumann approaches can be demonstrated, as done in Ref. [40] for acoustic problems. Hybrid methods are a powerful alternative to usual numerical approaches, transient ${ }^{41,42}$ or time-harmonic ${ }^{43,44}$, which requires longer FE meshes as well as modal post-processing steps of results.

In this paper, useful expressions are presented in order to calculate modal forces and power flows, and to efficiently separate ingoing from outgoing modes. These expressions may greatly simplify the development of hybrid methods inside commercial codes, as well as the post-process of power coefficients for the interpretation of results.

As illustrative examples, some results are finally shown on the scattering of the first extensional mode in free-end helical waveguides of different lay angles. Two validation tests are also performed in order to check the accuracy of the proposed approach. Though applied to elastic waves, the overall approach of this paper is rather general and may be applied or extended to other physics. 


\section{ON THE EXISTENCE OF GUIDED MODES IN CURVED WAVEGUIDES}

\section{A. Translational invariance}

By definition, guided modes (or propagation modes) are waves traveling without reflection along the axis of infinite waveguides. The time and axial evolution of such modes is

hence harmonic. In other words, an $e^{i(k s-\omega t)}$ dependence can be assumed and separated from all field components. In this paper, $k$ will denote the axial wavenumber, $s$ the axial coordinate and $\omega$ the angular frequency.

This axial dependence can only be applied if the considered waveguide is translationally invariant along its axis. Intuitively for straight waveguides, translational invariance means that both the cross-section shape and the material do not vary with respect to $s$. This intuitive definition is yet no longer accurate for curved waveguides. For instance, one may think of an axis corresponding to an arbitrary curve: waves can generally not travel without reflection because of the curvature variation, even though the section and the material remain invariant along $s$. Indeed, a third condition should be added in that case: the curvature of the axis should also remain constant.

From a mathematical point of view, the $e^{i k s}$ axial dependence indeed comes from a separation of variables that is applicable if the following fundamental property holds: the coefficients of equilibrium equations, including boundary conditions, must not depend on $s$. With this property, performing an axial Fourier transform do not give any convolution products but yields the same equilibrium equations as assuming an $e^{i k s}$ dependence: one can truly speak of propagation modes. This fundamental property is checked under the following three conditions:

1. the cross-section shape do not vary along the $s$-axis;

2. the material properties remain constant along $s$;

3. the coefficients associated with the partial derivatives of the differential operators that 
are involved in the physics under consideration (gradient, divergence, Laplacian,...) are independent on $s$.

These conditions somewhat extend the concept of translational invariance for curved waveguides. Condition 1 is geometric and necessary for the invariance of boundary conditions. Condition 2 is material. Both conditions are obvious and implicitly assumed in straight waveguide analyses. Condition 3 is non-trivial and closely related to the curvilinear coordinate system considered.

\section{B. Metric tensor}

Condition 3 may be highlighted thanks to the calculation of the metric tensor associated with the coordinate system. For a fundamental introduction to the use of general curvilinear coordinate systems, the reader may refer to Chapter 2 of Ref. [45] for instance.

Let us consider a curvilinear coordinate system denoted $(x, y, s), x$ and $y$ being the transverse coordinates. Let $(X, Y, Z)$ and $\left(\mathbf{e}_{X}, \mathbf{e}_{Y}, \mathbf{e}_{Z}\right)$ be the Cartesian coordinates and the Cartesian basis, respectively. Any position vector $(X, Y, Z)$ is uniquely related to $(x, y, s)$ through a mapping denoted $\boldsymbol{\Phi}$, as follows:

$$
X \mathbf{e}_{X}+Y \mathbf{e}_{Y}+Z \mathbf{e}_{Z}=\boldsymbol{\Phi}(x, y, s)
$$

In the following, the notation $(x, y, s)$ may be changed to $\left(x^{1}, x^{2}, x^{3}\right)$ for simplicity. The covariant basis is given by $\left(\mathbf{g}_{1}, \mathbf{g}_{2}, \mathbf{g}_{3}\right)$ where $\mathbf{g}_{i}=\partial \mathbf{\Phi} / \partial x^{i}(i=1,2,3)$. The contravariant basis, denoted $\left(\mathbf{g}^{1}, \mathbf{g}^{2}, \mathbf{g}^{3}\right)$, is defined from $\mathbf{g}^{i} \cdot \mathbf{g}_{j}=\delta_{j}^{i}$.

Then, the covariant metric tensor $\mathbf{g}$ is given by:

$$
g_{i j}=\mathbf{g}_{i} \cdot \mathbf{g}_{j}
$$

A fundamental point is that, when $\mathbf{g}$ does not depend on $s(\mathbf{g}=\mathbf{g}(x, y))$, condition 3 is automatically satisfied for any differential operator and hence, any physics. This can be explained by the fact that the coefficients of any differential operators are given by Christoffel 
symbols, which only depend on the $g_{i j}$ 's. For clarity, the Christoffel symbols $\Gamma_{i j}^{k}=\mathbf{g}_{i, j} \cdot \mathbf{g}^{k}$ are expressed as a function of the metric tensor as follows:

$$
\Gamma_{i j}^{k}=\frac{1}{2} g^{k l}\left(\frac{\partial g_{j l}}{\partial x^{i}}+\frac{\partial g_{i l}}{\partial x^{j}}-\frac{\partial g_{i j}}{\partial x^{l}}\right)
$$

where $g^{i j}=\mathbf{g}^{i} \cdot \mathbf{g}^{j}$ is the contravariant metric tensor, equal to the inverse of the covariant metric tensor $\left(g^{i j}=\left(\mathbf{g}^{-1}\right)_{i j}\right)$.

\section{Standard coordinate systems revisited}

For clarity, we first consider the cylindrical coordinate system $\left(x^{1}, x^{2}, x^{3}\right)=(r, \theta, z)$, obtained from the mapping $(X, Y, Z)=(r \cos \theta, r \sin \theta, z)$. Its metric tensor is:

$$
\mathbf{g}=\left[\begin{array}{lll}
1 & 0 & 0 \\
0 & r^{2} & 0 \\
0 & 0 & 1
\end{array}\right]
$$

As expected, $\mathrm{g}$ does not depend on $z$ (obvious existence of guided waves traveling in the z-direction). It does not depend on $\theta$ either (azimuthal direction), which also proves the existence of guided modes inside circular waveguides for any physics.

The spherical coordinate system $\left(x^{1}, x^{2}, x^{3}\right)=(r, \theta, \phi)$ is obtained from the mapping $(X, Y, Z)=(r \cos \theta \sin \phi, r \sin \theta \sin \phi, r \cos \phi) . \theta$ and $\phi$ are the azimuthal and zenith angles, respectively. The corresponding metric tensor is:

$$
\mathbf{g}=\left[\begin{array}{ccc}
1 & 0 & 0 \\
0 & r^{2} \sin ^{2} \phi & 0 \\
0 & 0 & r^{2}
\end{array}\right]
$$

$\mathrm{g}$ does not depend on $\theta$, which means that we can also speak of guided waves in the azimuthal direction for spherical waveguides (and any physics).

As mentioned in the introduction, analyses of circular and spherical waveguides have already been conducted (in elastodynamics). Yet, the existence of guided waves also occurs in some other coordinate systems. For instance, the metric tensor of spheroidal coordinate 
systems (oblate or prolate) does not depend on the azimuthal coordinate either. Also, helical coordinate systems are invariant along the helix axis, as shown in the next subsection.

\section{Helical coordinate system}

A helical coordinate system is now built. One starts by defining the helix centerline curve, described by the following position vector in the Cartesian orthonormal basis:

$$
\mathbf{R}(s)=R \cos \frac{2 \pi}{l} s \mathbf{e}_{X}+R \sin \frac{2 \pi}{l} s \mathbf{e}_{Y}+\frac{L}{l} s \mathbf{e}_{Z}
$$

where $l=\sqrt{L^{2}+4 \pi^{2} R^{2}}$ is the curvilinear length of one helix step. $R$ and $L$ are respectively the radius of the centerline in the $(X, Y)$ Cartesian plane and the helix step along the $Z$ axis (see Fig. 1). The helix lay angle $\phi$ is defined from: $\tan \phi=2 \pi R / L$. The unit tangent, normal and binormal vectors to the centerline are respectively obtained from $\mathbf{T}=d \mathbf{R} / d s$ and the Serret-Frenet formula ${ }^{46}: d \mathbf{T} / d s=-\kappa \mathbf{N}, d \mathbf{N} / d s=\tau \mathbf{B}+\kappa \mathbf{T}$ and $d \mathbf{B} / d s=-\tau \mathbf{N}$ (one has $\mathbf{B}=\mathbf{T} \wedge \mathbf{N}$ ). Note that $\mathbf{N}$ is oriented outward the curvature in this paper. For a helix, both the curvature $\kappa=4 \pi^{2} R / l^{2}$ and torsion $\tau=2 \pi L / l^{2}$ are constant.

For clarity, $\mathbf{N}, \mathbf{B}$ and $\mathbf{T}$ are expressed in the Cartesian basis as:

$$
\begin{gathered}
\mathbf{N}(s)=\cos \frac{2 \pi s}{l} \mathbf{e}_{X}+\sin \frac{2 \pi s}{l} \mathbf{e}_{Y}, \\
\mathbf{B}(s)=-\frac{L}{l} \sin \frac{2 \pi s}{l} \mathbf{e}_{X}+\frac{L}{l} \cos \frac{2 \pi s}{l} \mathbf{e}_{Y}-\frac{2 \pi R}{l} \mathbf{e}_{Z}, \\
\mathbf{T}(s)=-\frac{2 \pi R}{l} \sin \frac{2 \pi s}{l} \mathbf{e}_{X}+\frac{2 \pi R}{l} \cos \frac{2 \pi s}{l} \mathbf{e}_{Y}+\frac{L}{l} \mathbf{e}_{Z}
\end{gathered}
$$

A helical coordinate system can be built from the orthonormal basis $(\mathbf{N}, \mathbf{B}, \mathbf{T})$, with the following mapping $\boldsymbol{\Phi}$ :

$$
\boldsymbol{\Phi}(x, y, s)=\mathbf{R}(s)+x \mathbf{N}(s)+y \mathbf{B}(s)
$$

Using Serret-Frenet formula, one obtains the covariant basis (non-orthogonal):

$$
\begin{gathered}
\mathbf{g}_{1}=\mathbf{N}(s), \mathbf{g}_{2}=\mathbf{B}(s) \\
\mathbf{g}_{3}=-\tau y \mathbf{N}(s)+\tau x \mathbf{B}(s)+(1+\kappa x) \mathbf{T}(s) .
\end{gathered}
$$




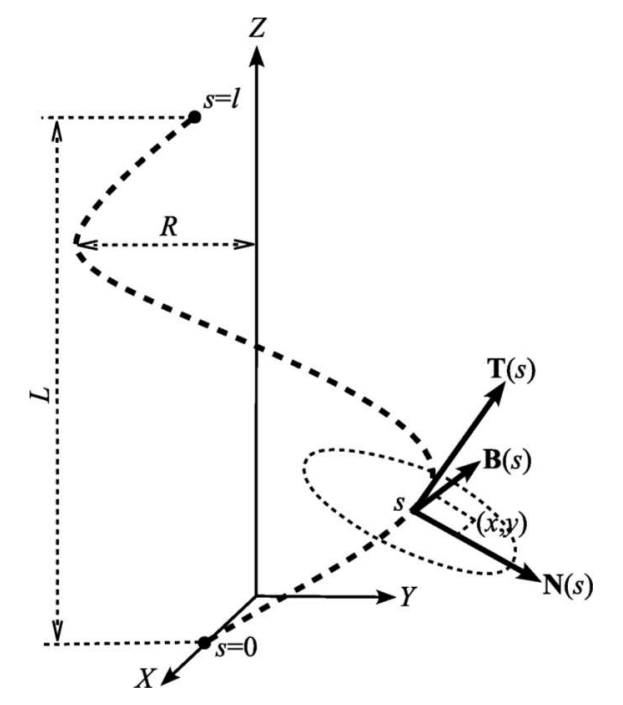

FIG. 1. Helix centerline curve (one step) with its associated Serret-Frenet basis and $(x, y, s)$ helical coordinate system.

and the calculation of the covariant metric tensor finally gives:

$$
\mathbf{g}=\left[\begin{array}{ccc}
1 & 0 & -\tau y \\
0 & 1 & \tau x \\
-\tau y & \tau x & \tau^{2}\left(x^{2}+y^{2}\right)+(1+\kappa x)^{2}
\end{array}\right]
$$

$\mathbf{g}$ does not depend on $s$. As a consequence, the coefficients of any partial differential operators expressed in the so-defined coordinate system are independent on $s$ too. This coordinate system can hence be used for the analysis of helical waveguides.

As a side remark, note that the following relations hold:

$$
R=\frac{\kappa}{\kappa^{2}+\tau^{2}}, L=\frac{2 \pi \tau}{\kappa^{2}+\tau^{2}}, \frac{2 \pi}{l}=\sqrt{\kappa^{2}+\tau^{2}}
$$

Then from Eqs. (6) and (7), it can be checked that the particular case $\tau=0$ degenerates into a circular coordinate system whose circular axis of radius $1 / \kappa$ lies into the $(X, Y)$ plane. The case $\kappa=0$ degenerates into a twisting system, as detailed in the next subsection. 


\section{E. Note on the twisting coordinate system}

A useful twisting coordinate system can be obtained from $\kappa=0$ and $\tau=2 \pi / L$. It corresponds to a system for which the $(x, y)$ plane (cross-section plane) rotates around and along the $Z$ axis $(s \equiv Z$ ) with an axial periodicity $L$ (rotation rate of $\tau=2 \pi / L$ ). The cross-section plane remains parallel to $(X, Y)$. Indeed, such a coordinate system also allows the analysis of a helical waveguide: in the local rotating $(x, y)$ plane, the cross-section of a helical waveguide do not change along $Z$ either (condition 1 is still satisfied).

Such a system is particularly interesting. It also allows the consideration of cylindrical waveguides of axis $Z$ for any value of $\tau$ ("a twisted cylinder remains a cylinder"). And as a consequence, a twisting system is suitable for the analysis of multi-wire waveguides (see Ref. [13]). This system will be chosen for the results obtained in the present paper. Note that this kind of system coincides with the one proposed in Ref. [9] for the analysis of pretwisted waveguides or in Ref. $[15,16]$ for electromagnetics.

Yet, the cross-section shape must be carefully considered because it is usually provided in the cutting plane normal to the helix centerline. With a twisting system, the shape must be determined in a plane parallel to the $(X, Y)$ plane. Let us find the cross-section cut by the plane $Z=0$. In the helical system, Eq. (8) yields:

$$
\left\{\begin{array}{l}
X=(R+x) \cos \frac{2 \pi s}{l}-y \frac{L}{l} \sin \frac{2 \pi s}{l} \\
Y=(R+x) \sin \frac{2 \pi s}{l}+y \frac{L}{l} \cos \frac{2 \pi s}{l} \\
Z=\frac{L}{l} s-\frac{2 \pi R}{l} y
\end{array}\right.
$$

so that $s=2 \pi y R / L$ at $Z=0$. Let $(x, y)=(x(t), y(t))$ be a curve parametrization of the cross-section normal to the helix centerline, $t$ denoting the curve parameter. Then, the cross-section cut by $Z=0$ can be parametrized as follows:

$$
\left\{\begin{array}{l}
X(t)=(R+x(t)) \cos (\alpha y(t))-y(t) \frac{L}{l} \sin (\alpha y(t)) \\
Y(t)=(R+x(t)) \sin (\alpha y(t))+y(t) \frac{L}{l} \cos (\alpha y(t))
\end{array}\right.
$$

where $\alpha=4 \pi^{2} R / l L$. The above parametrization yields the adequate cross-section that must be used with a twisting coordinate system. 

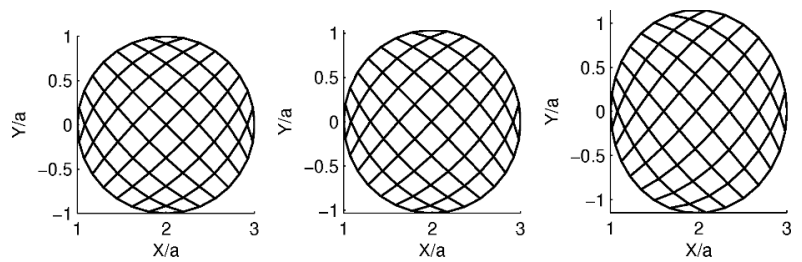

FIG. 2. $Z=0$ plane-cut of a helical waveguide $R=2 a$ having a circular cross-section of radius $a$ for the following lay angles: $\phi=0^{\circ}$ (cylinder), $15^{\circ}$ and $30^{\circ}$ (from left to right). The SAFE mesh used for the computations is also shown.

For a helical waveguide of circular section of radius $a$, one has $(x(t), y(t))=$ $(a \cos t, a \sin t)$ with $t \in[0 ; 2 \pi]$. Fig. 2 exhibits cross-section shapes obtained for $\phi=0,15$ and $30^{\circ}$ with $R=2 a$.

\section{ELASTIC GUIDED MODES IN HELICAL WAVEGUIDES}

In this section, elastic guided modes in helical waveguides are solved numerically. One assumes a linearly elastic material, small strains and displacements, with a time harmonic $e^{-i \omega t}$ dependence. The general approach is based on a SAFE technique, which is briefly recalled. No special assumption is required on waveguide properties: arbitrary cross-sections, heterogeneous or anisotropic materials are allowed (but with invariance along the axis).

First, one recalls the variational formulation of elastodynamics for clarity:

$$
\int_{\Omega} \delta \boldsymbol{\epsilon}^{T} \boldsymbol{\sigma} d V-\omega^{2} \int_{\Omega} \rho \delta \mathbf{u}^{T} \mathbf{u} d V=\int_{\Omega} \delta \mathbf{u}^{T} \mathbf{q} d V+\int_{\delta \Omega} \delta \mathbf{u}^{T} \mathbf{f} d V
$$

where $\mathbf{u}, \boldsymbol{\epsilon}$ and $\boldsymbol{\sigma}$ are the displacement strain and stress vectors. $\delta$ denotes virtual fields. $\mathbf{f}$ is the traction vector on the surface $\delta \Omega . \rho$ is the material density and $\mathbf{q}$ represents some known acoustic sources inside the structural volume $\Omega$. 


\section{A. Strain-displacement and Stress-strain relations}

In a general non-orthogonal curvilinear coordinate system, the strain-displacement relationship is expressed as:

$$
\epsilon_{i j}=1 / 2\left(u_{i, j}+u_{j, i}\right)-\Gamma_{i j}^{k} u_{k}
$$

where subscripts denote covariant components with respect to the contravariant basis. The notation $(\cdot)_{, i}(i=1,2,3)$ is used for derivatives with respect to $x, y$ and $s$ respectively. Note that covariant components generally have non-physical units and the contravariant basis is non-orthogonal. In the remainder, physical components with respect to the orthonormal Serret-Frenet basis $(\mathbf{N}, \mathbf{B}, \mathbf{T})$ will be preferred.

The strain-displacement relation can be rewritten as follows:

$$
\boldsymbol{\epsilon}=\left(\mathbf{L}_{x y}+\mathbf{L}_{s} \partial / \partial s\right) \mathbf{u}
$$

where $\mathbf{L}_{x y}$ is the operator containing all terms except the derivatives with respect to the $s$-axis. $\mathbf{u}=\left[\begin{array}{lll}u_{n} & u_{b} & u_{t}\end{array}\right]^{T}$ is the displacement field, where the superscript $T$ denotes the matrix

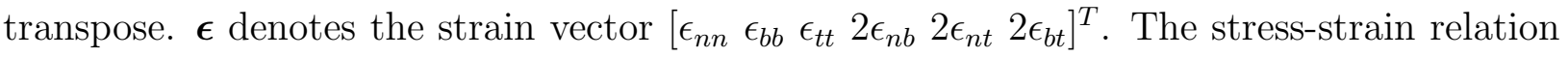
is then expressed as:

$$
\sigma=\mathrm{C} \epsilon
$$

where $\boldsymbol{\sigma}=\left[\begin{array}{llllll}\sigma_{n n} & \sigma_{b b} & \sigma_{t t} & \sigma_{n b} & \sigma_{n t} & \sigma_{b t}\end{array}\right]^{T}$ is the stress vector and $\mathbf{C}$ is the matrix of material properties, expressed in the orthonormal Serret-Frenet basis. C might be complex (as it is the case for viscoelastic materials).

With the helical coordinate system of Sec. II.D, the expression of $\mathbf{L}_{x y}$ and $\mathbf{L}_{s}$ are given by: 


$$
\mathbf{L}_{x y}=\frac{1}{1+\kappa x}\left[\begin{array}{ccc}
(1+\kappa x) \partial / \partial x & 0 & 0 \\
0 & (1+\kappa x) \partial / \partial y & 0 \\
\kappa & 0 & \tau y \partial / \partial x-\tau x \partial / \partial y \\
\tau y \partial / \partial x-\tau x \partial / \partial y & -\tau & -\kappa+(1+\kappa x) \partial / \partial x \\
\tau & \tau y \partial / \partial x-\tau x \partial / \partial y & (1+\kappa x) \partial / \partial y
\end{array}\right], \quad \mathbf{L}_{s}=\frac{1}{1+\kappa x}\left[\begin{array}{lll}
0 & 0 & 0 \\
0 & 0 & 0 \\
0 & 0 & 1 \\
0 & 0 & 0 \\
1 & 0 & 0 \\
0 & 1 & 0
\end{array}\right]
$$

Details of calculations can be found in Refs. [13, 31]. As expected, note that both operators do not depend on $s$ so that we can truly assume and separate the axial $e^{i k s}$ dependence.

\section{B. SAFE formulation}

There is no external force for the purpose of studying propagation modes. The SAFE technique consist in assuming an $e^{i k s}$ dependence for $\mathbf{u}\left(e^{-i k s}\right.$ for $\left.\delta \mathbf{u}\right)$ in the variational formulation (14) before FE discretization. Then, $\partial / \partial s$ can be replaced by $i k$ and the exponential separated from all field components. The dimensionality of the problem is hence reduced to the two dimensions of the cross-section.

From Eqs. (14), (16) and (17), this leads to the following eigenvalue problem for the determination of guided modes ${ }^{13,31}$ :

$$
\left\{\mathbf{K}_{1}-\omega^{2} \mathbf{M}_{S}+i k\left(\mathbf{K}_{2}-\mathbf{K}_{2}^{T}\right)+k^{2} \mathbf{K}_{3}\right\} \mathbf{U}=\mathbf{0}
$$

with the following elementary matrices:

$$
\begin{gathered}
\mathbf{K}_{1}^{e}=\int_{S^{e}} \mathbf{N}^{e T} \mathbf{L}_{x y}^{T} \mathbf{C L}_{x y} \mathbf{N}^{e} \sqrt{g} d S, \\
\mathbf{K}_{2}^{e}=\int_{S^{e}} \mathbf{N}^{e T} \mathbf{L}_{x y}^{T} \mathbf{C} \mathbf{L}_{s} \mathbf{N}^{e} \sqrt{g} d S, \\
\mathbf{K}_{3}^{e}=\int_{S^{e}} \mathbf{N}^{e T} \mathbf{L}_{s}^{T} \mathbf{C} \mathbf{L}_{s} \mathbf{N}^{e} \sqrt{g} d S, \\
\mathbf{M}_{S}^{e}=\int_{S^{e}} \rho \mathbf{N}^{e T} \mathbf{N}^{e} \sqrt{g} d S
\end{gathered}
$$

$\mathbf{U}$ is the column vector containing nodal displacements expressed in the orthonormal SerretFrenet basis. $\rho$ is the material density. $\quad \mathbf{N}^{e}$ is a matrix of nodal interpolating functions 
of displacement on the element, $d S=d x d y$ and $g$ is the determinant of the metric tensor $(g=\operatorname{det} \mathbf{g})$.

Due to the symmetry of $\mathbf{K}_{1}, \mathbf{K}_{3}$ and $\mathbf{M}_{S}$, the eigenproblem has two sets of eigensolutions $\left(k_{n}^{+}, \mathbf{U}_{n}^{+}\right)$and $\left(-k_{n}^{+}, \mathbf{U}_{n}^{-}\right)(n=1, \ldots, N)$, representing $N$ positive-going and $N$ negative-going wave types ( $N$ being the number of degrees of freedom (dofs)).

Given $\omega$ and finding $k$, this eigenproblem is quadratic. It is recast into a generalized linear eigensystem written for $\left[\mathbf{U}^{T} k \mathbf{U}^{T}\right]^{T}$ in order to be solved by standard numerical solvers $^{31}$ - see Ref. [47] for details.

\section{Mode properties}

Once the eigenproblem has been solved, the calculation of modal properties is crucial for the analysis and interpretation of solutions.

Let $\left(k_{n}, \mathbf{U}_{n}\right)$ be a given eigenmode, positive or negative-going (the \pm sign is dropped for conciseness of notations). The phase velocity $v_{p_{n}}$ and the attenuation $\beta_{n}$ are simply given by $v_{p_{n}}=\omega / \Re\left(k_{n}\right)$ and $\beta_{n}=\Im\left(k_{n}\right)$, respectively.

The kinetic and potential energies are respectively defined as $E_{k}=\frac{1}{2} \rho v_{\alpha} v_{\alpha}$ and $E_{p}=$ $\frac{1}{2} \epsilon_{\alpha \beta} \sigma_{\alpha \beta}(\alpha=n, b, t)$, where $v_{\alpha}=\dot{u}_{\alpha}$ is the velocity vector. The cross-section and time averaged energies of mode $n$ can be obtained from:

$$
\begin{gathered}
\int_{S} \bar{E}_{k_{n}} d S=\frac{\omega^{2}}{4} \Re\left(\mathbf{U}_{n}^{T *} \mathbf{M}_{S} \mathbf{U}_{n}\right), \\
\int_{S} \bar{E}_{p_{n}} d S=\frac{1}{4} \Re\left\{\mathbf{U}_{n}^{T *}\left(\mathbf{K}_{1}+i k_{n}\left(\mathbf{K}_{2}-\mathbf{K}_{2}^{T}\right)+k_{n}^{2} \mathbf{K}_{3}\right) \mathbf{U}_{n}\right\} .
\end{gathered}
$$

where bars denote time averaging and the superscript ${ }^{*}$ refers to the complex conjugate. From Eq. (19), note that each mode $n$ satisfies the equality $\int_{S} \bar{E}_{k_{n}} d S=\int_{S} \bar{E}_{p_{n}} d S$.

The post-process of modal power flow and energy velocity is less straightforward. This paper gives some explicit formula, derived below.

The complex Poynting vector ${ }^{48} \mathbf{P}$, defined in analogy with the electromagnetic case, is given by: $P_{\beta}=-\frac{1}{2} v_{\alpha}^{*} \sigma_{\alpha \beta}$. The complex power flow (cross-section averaged) is: $\Pi=$ $\int_{S} \mathbf{P} \cdot \mathbf{n} d S$, where $\mathbf{n}$ is the unit vector along the propagation direction (normal to the cross- 
section). Provided that $\mathbf{v}=-i \omega \mathbf{u}$ and $\mathbf{n}=\mathbf{T}$, one has:

$$
\mathbf{P} \cdot \mathbf{n} d S=-\frac{i \omega}{2} u_{\alpha}^{*} \sigma_{\alpha t} d S
$$

Then, it can be checked that $u_{\alpha}^{*} \sigma_{\alpha t}=\mathbf{u}^{T *} \mathbf{L}_{s}^{T} \boldsymbol{\sigma}(1+\kappa x)=\mathbf{u}^{T *} \mathbf{L}_{s}^{T} \mathbf{C}\left(\mathbf{L}_{x y}+i k \mathbf{L}_{s}\right) \mathbf{u}(1+\kappa x)$, so that the following useful expression holds for the complex power flow of mode $n$ (cross-section averaged):

$$
\Pi_{n}=-\frac{i \omega}{2} \mathbf{U}_{n}^{T *}\left(\mathbf{K}_{2}^{T}+i k_{n} \mathbf{K}_{3}\right) \mathbf{U}_{n}
$$

The real part $\Re\left(\Pi_{n}\right)$ coincides with the cross-section and time averaged power flow.

Then, the cross-section and time averaged energy velocity in waveguides is defined as follows ${ }^{49}$ :

$$
v_{e_{n}}=\frac{\Re\left(\Pi_{n}\right)}{\int_{S}\left(\bar{E}_{k_{n}}+\bar{E}_{p_{n}}\right) d S}
$$

Expressions (21) and (23) allow a direct computation of the energy velocity. The energy velocity is an important wave property that remains appropriate even for damped media ${ }^{50}$ (as opposed to the group velocity definition $v_{g_{n}}=\partial \omega / \partial k_{n}$, which is generally not valid for damped waveguides).

Modes can be normalized with respect to the mass matrix, yielding $\mathbf{U}_{n}^{T *} \mathbf{M}_{S} \mathbf{U}_{n}=1$. Since the eigenvectors may be multiplied by any complex scalar of unit modulus, such a normalization is not unique, which might be not convenient in practice (for instance, when we want to track modes by visual inspection of their modeshapes). The above normalization can be made unique with the additional requirement: for a given $n, \mathbf{U}_{n}$ is multiplied by a complex scalar of unit modulus, such that its degree of freedom of maximum modulus is made real and positive.

\section{Results}

We consider waveguides of circular cross-section of radius a (in the plane normal to the helix centerline). For conciseness, we briefly give results for helical waveguides of helix radius $R=2 a$ and the following three lay angles: $\phi=0^{\circ}$ (cylinder), $15^{\circ}$ and $30^{\circ}$. These 
three cases will also be considered in Sec. IV. For more detailed physical interpretations and parametric studies on helical waveguides, the reader can refer to $[12,31]$. The validation of the proposed SAFE method has been performed in Ref. [31] with a helical coordinate system, and in Ref. [13] with a twisting system.

Without loss of generality, no material damping is considered and the material is assumed to be isotropic. The Poisson coefficient is $\nu=0.25$. The shear velocity is defined as $v_{s}=\sqrt{E / 2 \rho(1+\nu)}$, where $E$ denotes Young modulus. Variables are made dimensionless with characteristic length and time, chosen as $a$ and $a / c_{s}$, respectively. The dimensionless frequency is then given by $\omega a / c_{s}$. As already mentioned, a twisting system is used and the SAFE meshes are given by Fig. 2. The geometry is meshed with 8-node quadrangles (quadratic elements are used), yielding 675 dofs. SAFE computations are held at fixed dimensionless frequency $\omega a / c_{s} \in[0 ; 5]$, discretized into 250 equal steps.

Figure 3 shows the dimensionless energy velocity vs. frequency plots. The results obtained for $\phi=0^{\circ}$ corresponds to a straight cylindrical waveguide, for which solutions are well-known in the literature. For that case, modes have been labeled accordingly to the convention used by Meitzler ${ }^{51}$. The fastest mode in the low frequency range $\left(\omega a / c_{s} \leqslant 2\right)$, which is colored gray on the plots, corresponds to the first compressional-like mode (referred to as the $L(0,1)$ mode in cylinders). Though somewhat subjective, a visual inspection of modeshapes has been done in order to identify the evolution of this mode on a wider frequency range.

As expected, differences between cylinder and helical waveguides become greater as the lay angle increases. The energy velocity of the $L(0,1)$-like mode decays as the lay angle increases, which is essentially due to the lengthening of the path covered by waves (waves travels along the helix axis but the wavenumber and velocity are measured along the $Z$ axis). In helical waveguides, note that this mode has a low but non-zero cut-off frequency that increases with the angle ${ }^{13,31}$.

Unlike in the cylindrical case, helical waveguides may exhibit sharp changes in dispersion curves at certain frequencies - see for instance the $F(1,2)$-like mode for $\phi=15^{\circ}$ near 

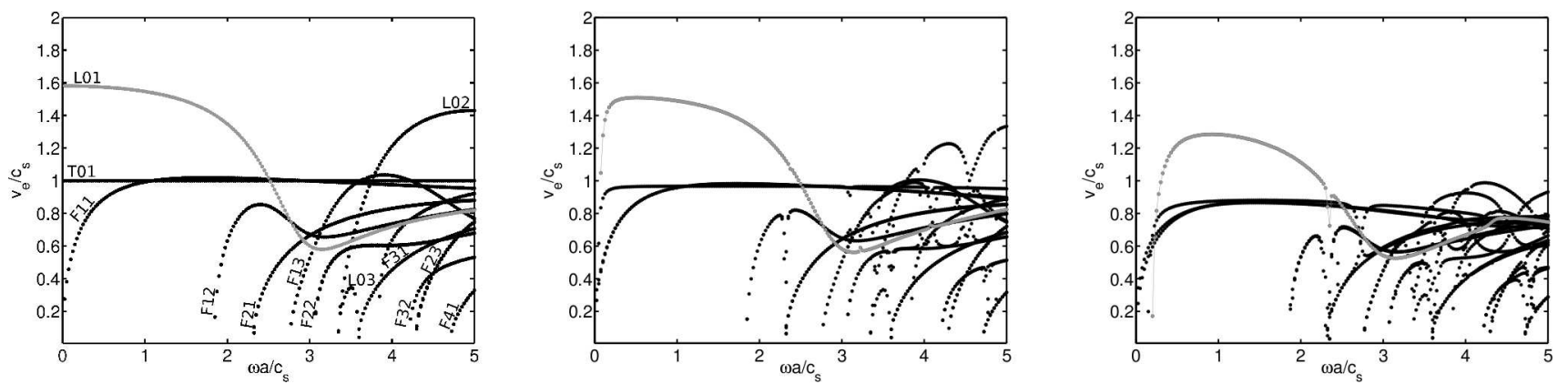

FIG. 3. From left to right: dimensionless energy velocity vs. frequency for $\phi=0^{\circ}$ (cylinder), $15^{\circ}$ and $30^{\circ}$. The first compressional-like mode is colored gray.

$\omega a / c_{s}=2.3$. These changes usually corresponds to curve veering or branch connection between different modes ${ }^{13,31}$.

\section{MODE SCATTERING BY INHOMOGENEITIES}

Based on the previously obtained eigensolutions, a three-dimensional hybrid FE-SAFE method is developed for the analysis of mode scattering by inhomogeneities inside helical waveguides. Inhomogeneities are assumed to be local (restricted to a small portion of the waveguide) but can be of complex shape. The usual FE method is applied to a region of the waveguide including the inhomogeneity (a crack for instance). Then, SAFE modal expansions are applied at cross-section boundaries of the three-dimensional FE model, where semi-infinite waveguides are to be connected. This technique yields transparent conditions.

For simplicity, Fig. 4 depicts the FE portion, denoted $\Omega$, of a free-end waveguide. The free-end is on the top. The reflection of waves inside a free-end waveguide is a typical scattering problem. A free-end boundary can also be viewed as a complete crack of the wire. Incident waves travel from the bottom to the top, reflected waves by the free-end travel in the opposite direction. The bottom cross-section, denoted $\Sigma$, corresponds to the boundary where a semi-infinite waveguide is to be connected. The boundary condition on $\Sigma$ should be such that modes reflected by the free-end go out from $\Omega$ without reflecting on $\Sigma$ (transparent boundary). 

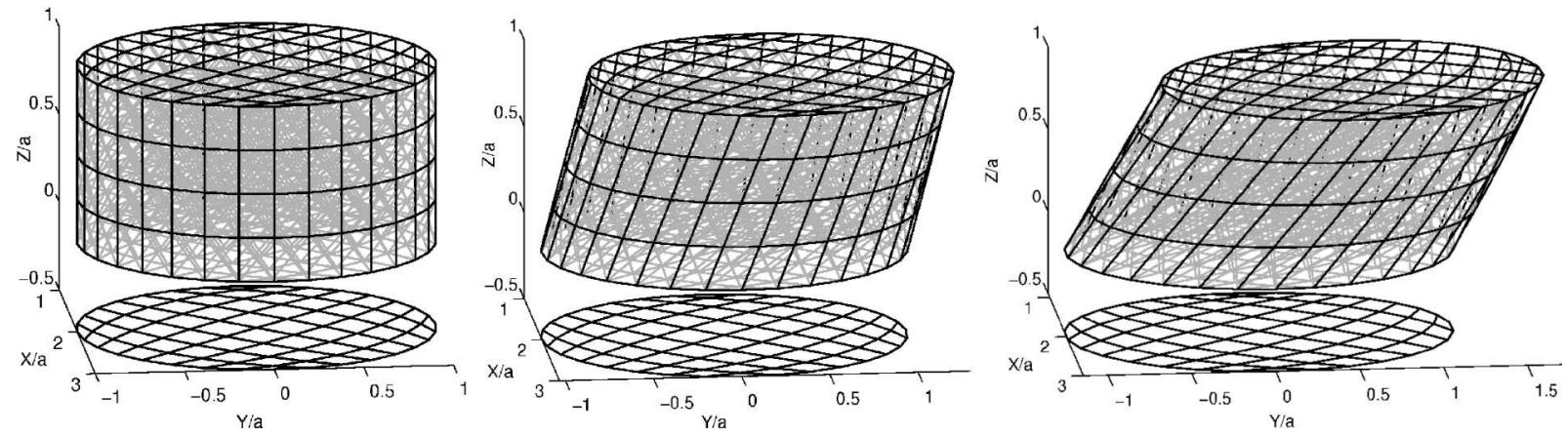

FIG. 4. Geometry and FE mesh used for the computations with the hybrid method for a free-end helical waveguide with $R=2 a$ and the following lay angles: $\phi=0^{\circ}$ (cylinder), $15^{\circ}$ and $30^{\circ}$ (from left to right). The bottom cross-section, here duplicated in the plane $Z=-0.5$, corresponds to $\Sigma$ (transparent condition). The remaining boundaries of the FE model are free, including the top cross-section representing the free-end.

\section{A. Hybrid FE-SAFE formulation}

We start from the FE model of the region $\Omega$. The discretization of the variational formulation (14) leads to the usual FE system for three-dimensional elastodynamics:

$$
\left(\mathbf{K}-\omega^{2} \mathbf{M}\right) \mathbf{U}=\mathbf{F}
$$

where $\mathbf{K}$ and $\mathbf{M}$ are the stiffness and mass matrices. $\mathbf{U}$ is the vector of displacement dofs of the whole FE region.

This system is partitioned into dofs associated with the cross-section $\Sigma$ and the remaining dofs, as follows:

$$
\mathbf{U}=\left\{\begin{array}{l}
\mathbf{U}_{\Sigma} \\
\mathbf{U}_{\bar{\Sigma}}
\end{array}\right\}, \quad \mathbf{F}=\left\{\begin{array}{l}
\mathbf{F}_{\Sigma} \\
\mathbf{F}_{\bar{\Sigma}}
\end{array}\right\}
$$

with $\bar{\Sigma}=\Omega \backslash \Sigma$.

The basic idea of hybrid methods consists in expanding both $\mathbf{U}_{\Sigma}$ and $\mathbf{F}_{\Sigma}$ on the guided modes associated with the cross-section $\Sigma$ :

$$
\begin{aligned}
\mathbf{U}_{\Sigma} & =\sum_{n=1}^{N} \alpha_{n}^{-} \mathbf{U}_{n}^{-}+\sum_{n=1}^{N} \alpha_{n}^{+} \mathbf{U}_{n}^{+}, \\
\mathbf{F}_{\Sigma} & =\sum_{n=1}^{N} \alpha_{n}^{-} \mathbf{F}_{n}^{-}+\sum_{n=1}^{N} \alpha_{n}^{+} \mathbf{F}_{n}^{+}
\end{aligned}
$$


$\mathbf{U}_{n}^{ \pm}$denote the displacement modeshape of mode $n$, obtained from SAFE analysis. $\mathbf{F}_{n}^{ \pm}$is the associated modal force (calculation detailed in the next subsection). The $\alpha_{n}^{ \pm}$'s are modal coefficients. Ingoing modes are differentiated from outgoing modes thanks to the superscripts - and + respectively. In the present example, ingoing modes are incident modes while outgoing modes are reflected modes. $N$ now denotes the number of modes retained in the expansion, and after truncation, is lower than the number of degree of freedom.

The incident coefficients $\alpha_{n}^{-}$'s are known. The scattered coefficients $\alpha_{n}^{+}$'s are unknowns and must be solved. A convenient way of rewriting the expansions (27) is to use global matrix forms. First, we separate and gather the unknown variables into a single global vector $\mathbf{U}^{+}$as well as the known variables into a vector $\mathbf{U}^{-}$, defined as follows:

$$
\mathrm{U}^{-}=\left\{\begin{array}{l}
\boldsymbol{\alpha}^{-} \\
\mathrm{F}_{\bar{\Sigma}}
\end{array}\right\}, \quad \mathrm{U}^{+}=\left\{\begin{array}{l}
\boldsymbol{\alpha}^{+} \\
\mathrm{U}_{\bar{\Sigma}}
\end{array}\right\}
$$

where $\boldsymbol{\alpha}^{ \pm}$are the modal coefficients stored in column vectors. Note that $\mathbf{F}_{\bar{\Sigma}}$ is known and may represent acoustic sources located inside the FE region (but outside $\Sigma$ ).

Then, the eigenmode expansions (27) can be rewritten into the following global forms:

$$
\mathbf{U}=\mathbf{G}_{u}^{-} \mathbf{U}^{-}+\mathbf{G}_{u}^{+} \mathbf{U}^{+}, \quad \mathbf{F}=\mathbf{G}_{f}^{-} \mathbf{U}^{-}+\mathbf{G}_{f}^{+} \mathbf{U}^{+}
$$

with the following notations:

$$
\begin{aligned}
& \mathbf{G}_{u}^{-}=\left[\begin{array}{cc}
\mathbf{B}_{u}^{-} & \mathbf{0} \\
\mathbf{0} & \mathbf{0}
\end{array}\right], \\
& \mathbf{G}_{f}^{-}=\left[\begin{array}{cc}
\mathbf{B}_{f}^{-} & \mathbf{0} \\
\mathbf{0} & \mathbf{I}
\end{array}\right],
\end{aligned}
$$

where $\mathbf{B}_{u}^{ \pm}$and $\mathbf{B}_{f}^{ \pm}$denote the modal basis of eigendisplacements and eigenforces, stored in column:

$$
\mathbf{B}_{u}^{ \pm}=\left[\mathbf{U}_{1}^{ \pm}\left|\mathbf{U}_{2}^{ \pm}\right| \ldots \mid \mathbf{U}_{N}^{ \pm}\right], \quad \mathbf{B}_{f}^{ \pm}=\left[\mathbf{F}_{1}^{ \pm}\left|\mathbf{F}_{2}^{ \pm}\right| \ldots \mid \mathbf{F}_{N}^{ \pm}\right]
$$

Finally, using Eq. (29) into Eq. (25) yields a linear matrix system for $\mathbf{U}^{+}$, which is inverted at each frequency $\omega$ :

$$
\mathbf{G}_{u}^{+T}\left(\mathbf{D G}_{u}^{+}-\mathbf{G}_{f}^{+}\right) \mathbf{U}^{+}=\mathbf{G}_{u}^{+T}\left(\mathbf{G}_{f}^{-}-\mathbf{D G}_{u}^{-}\right) \mathbf{U}^{-}
$$


$\mathbf{D}=\mathbf{K}-\omega^{2} \mathbf{M}$ is the initial dynamic stiffness of the FE problem. Physical solutions can be reconstructed from Eq. (29). The procedure can be readily extended to the case of two transparent boundaries (or more), as it is the case for reflection-transmission problems in infinite waveguides (see IV.E.1).

Note that the scattered modal coefficients are directly solved for, which might be advantageous compared to other methods requiring additional post-processing steps ${ }^{43,52}$. Besides, $\mathbf{U}_{\bar{\Sigma}}$ is not condensed out in order to avoid the inversion of the dynamic stiffness (computationally expensive for three-dimensional problems).

\section{B. Modal forces}

With standard SAFE methods, the calculation of the modal forces $\mathbf{F}_{n}^{ \pm}$from the modal displacements $\mathbf{U}_{n}^{ \pm}$is a crucial point. Ideally, this calculation should be consistent with the FE approximation used. Nodal forces can be obtained by post-processing load vectors from displacement derivatives ${ }^{35,38}$ associated to each mode, but this procedure might be tedious and time-consuming in practice. An explicit formula can indeed be obtained, as shown in the following.

$\mathbf{F}_{\Sigma}$ is the left-hand side of Eq. (25) restricted to $S=\Sigma$ and comes from the variational form $\int_{\Sigma} \delta \mathbf{u}^{T} \mathbf{f} d S=\delta \mathbf{U}_{\Sigma}^{T} \mathbf{F}_{\Sigma}$, where $f_{\alpha}=\sigma_{\alpha \beta} n_{\beta}$ is the external traction ( $\mathbf{n}$ is the outward unit normal). The normal mode expansion gives $\delta \mathbf{U}_{\Sigma}^{T} \mathbf{F}_{\Sigma}=\sum_{n=1}^{N} \alpha_{n}^{-} \delta \mathbf{U}_{\Sigma}^{T} \mathbf{F}_{n}^{-}+\sum_{n=1}^{N} \alpha_{n}^{+} \delta \mathbf{U}_{\Sigma}^{T} \mathbf{F}_{n}^{+}$, where the $\mathbf{F}_{n}^{ \pm}$'s can be defined from:

$$
\delta \mathbf{U}_{\Sigma}^{T} \mathbf{F}_{n}^{ \pm}=\int_{\Sigma} \delta \mathbf{u} \cdot \mathbf{f}_{n}^{ \pm} d S
$$

Note that $\mathbf{n}=\mathbf{T}$ on $\Sigma$. Similarly to the power flow in Sec. III.C, we can write: $\mathbf{f}_{n}^{ \pm}=$ $\mathbf{L}_{s}^{T} \boldsymbol{\sigma}_{n}^{ \pm}(1+\kappa x)=\mathbf{L}_{s}^{T} \mathbf{C}\left(\mathbf{L}_{x y}+i k_{n}^{ \pm} \mathbf{L}_{s}\right) \mathbf{u}_{n}^{ \pm}(1+\kappa x)$. From this expression and Eq. (33), it can be checked that the modal forces are explicitly given by:

$$
\mathbf{F}_{n}^{ \pm}=\left(\mathbf{K}_{2}^{T}+i k_{n}^{ \pm} \mathbf{K}_{3}\right) \mathbf{U}_{n}^{ \pm}
$$

As a side remark, it should be noted that the system (32) is in fact symmetric. This is 
due to the following property:

$$
\left(k_{n}+k_{m}\right)\left(\mathbf{U}_{m}^{T} \mathbf{F}_{n}-\mathbf{U}_{n}^{T} \mathbf{F}_{m}\right)=0
$$

which could be viewed as the discretized form of the real biorthogonality relationship ${ }^{53}$. This relation is obtained by subtracting the SAFE system (19) written for $\mathbf{U}_{n}$ and pre-multiplied by $\mathbf{U}_{m}^{T}$, from the transpose of the system written for $\mathbf{U}_{m}$ and pre-multiplied by $\mathbf{U}_{n}^{T}$. Note that the \pm signs have been dropped because the traveling direction is not considered in the above relation. Let us consider outgoing-modes only. Then, $k_{n}^{+}+k_{m}^{+} \neq 0$ so that

$\mathbf{U}_{m}^{+T} \mathbf{F}_{n}^{+}=\mathbf{U}_{n}^{+T} \mathbf{F}_{m}^{+}(\forall m, n)$, which proves that $\mathbf{B}_{u}^{+T} \mathbf{B}_{f}^{+}$is symmetric. As a consequence, the matrix $\mathbf{G}_{u}^{+T}\left(\mathbf{D G}_{u}^{+}-\mathbf{G}_{f}^{+}\right)$is also symmetric. Note that this property still holds in the damped case, if $\mathbf{C}$ is complex (case of viscoelastic material).

\section{Traveling direction}

Another difficulty concerns the determination of traveling direction for each mode. In undamped waveguides ( $\mathbf{C}$ is real), eigensolutions for which $k_{n}$ is purely real, purely imaginary and fully complex represent propagating waves, evanescent waves and inhomogeneous waves (decaying but oscillatory), respectively. Furthermore, backward modes might occur in elastic waveguides (the energy and phase velocities of such modes have opposite signs).

Consequently, positive-going modes should be separated from negative-going ones by the criterion $v_{e_{n}}>0$ when propagating, and by $\Im\left(k_{n}\right)>0$ when non-propagating. The problem consists in distinguishing propagating from non-propagating modes.

In undamped waveguides, propagating and non-propagating modes respectively satisfy $\Im\left(\Pi_{n}\right)=0$ and $\Re\left(\Pi_{n}\right)=0$. Hence in practice, the condition $\left|\Re\left(\Pi_{n}\right)\right|>\left|\Im\left(\Pi_{n}\right)\right|$ can be proposed as an efficient criterion for finding propagating modes. In damped waveguides, $\Pi_{n}$ usually becomes fully complex. Nevertheless, quasi-propagating modes are expected to have a small imaginary part so that the criterion can still be applicable. 


\section{Results for free-end elastic helical waveguides}

As illustrative examples, we analyze modal scattering occurring in semi-infinite waveguides terminated by a free-end. The same helix parameters as in Sec. III.D are considered $\left(R=2 a, \phi=0,15\right.$ and $\left.30^{\circ}\right)$. A single mode is incident, corresponding to the $L(0,1)$-like mode previously identified on the dispersion curves (Fig. 3).

It is noteworthy that the modal expansion on $\Sigma$ also accounts for non-propagating modes, which allows to set the free-end boundary relatively close to the boundary $\Sigma$. In the present paper, the latter is set at $Z=0$ while the former is at $Z=a$. Hybrid FE-SAFE meshes are shown in Fig. 4 (for clarity, $\Sigma$ is duplicated at $Z=-a / 2$, but must be understood at $Z=a$ ). 20-node quadratic brick elements have been used, yielding 4347 dofs.

As already mentioned, guided modes are computed with a twisted system. This has the advantage of avoiding any transformation between Cartesian and Serret-Frenet components, because the $\Sigma$ plane coincides with the Cartesian $(X, Y)$ plane.

In the following computations, modal bases are truncated by retaining modes satisfying $|\Im(k a)| \leqslant 5$. A good accuracy is expected because the amplitudes of non-propagating modes reflected from the free end, but not taken into account in the expansion, would then be divided by at least $e^{5} \simeq 148$ at the transparent boundary $\Sigma$. In practice, the error on the power balance has been checked at each frequency and for the three waveguides. This error can be defined as:

$$
e=\frac{\Re\left(\Pi^{-}\right)+\Re\left(\Pi^{+}\right)}{\Re\left(\Pi^{-}\right)}
$$

where $\Pi^{ \pm}=-i \omega\left(\mathbf{G}_{u}^{ \pm} \mathbf{U}^{ \pm}\right)^{T *}\left(\mathbf{G}_{f}^{ \pm} \mathbf{U}^{ \pm}\right) / 2$ is the total complex power flow of $-/+$ modes (ingoing/outgoing). In the following results, the worst error has been found to be less that $0.8 \%$, which is quite satisfying. This tends to confirm that the proposed truncation does not yield any significant loss of accuracy.

Figure 5 shows the power reflection coefficients vs. frequency plots obtained for the three waveguides, where the power reflection coefficient is defined as:

$$
R_{n}=\frac{\Re\left(\Pi_{n}^{+}\right)}{\Re\left(\Pi_{i}^{-}\right)}
$$



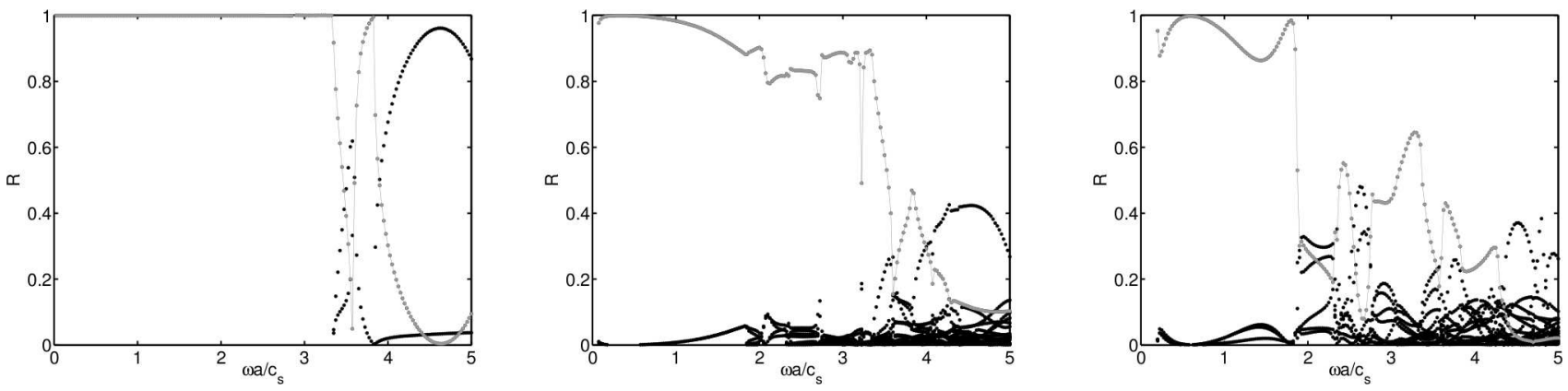

FIG. 5. From left to right: power reflection coefficient vs. frequency for $\phi=0^{\circ}$ (cylinder), $15^{\circ}$ and $30^{\circ}$. The first compressional-like mode is colored gray.

where the index $i$ denotes the incident mode. In the cylinder case $\left(\phi=0^{\circ}\right)$, the $L(0,1)$ mode is fully reflected until $\omega a / c_{s} \simeq 3.3$. This frequency is indeed the first cut-off frequency of higher compressional modes, corresponding to $L(0,2)$ and $L(0,3)$. Above that frequency, the sudden drop of the $L(0,1)$ reflection indicates that this mode is partially converted to $L(0,2)$ and $L(0,3)$. A full conversion might even occur at certain frequencies. The free-end cylinder case is further considered in Sec. IV.E.2, where a comparison with literature results is given.

The geometry of a free-end cylinder is fully axisymmetric. Besides, compressional $L(0, n)$ modes are axisymmetric, unlike flexural modes $F(m, n)$. As a consequence, there is no conversion of the $L(0,1)$ to $F(m, n)$ modes. In the case of helical waveguides $\left(\phi \neq 0^{\circ}\right)$, this symmetry is broken and conversion to flexural or torsional modes occurs. This causes a decrease of the power reflection coefficient of the $L(0,1)$-like mode in the lowest frequency range, far below the first cut-off frequencies of $L(0, n)$-like modes. As usual with modal scattering in waveguides ${ }^{35,38}$, the sharp changes of the reflection coefficient generally coincide with cut-off frequencies (compare Figs. 3 and 5).

For the angle of $15^{\circ}$, some rough similarities may still be found with the cylinder case. For instance, a strong reflection drop is also observed near 3.3, which is the cut-off frequencies of the $L(0,2)$ and $L(0,3)$-like mode. However for $30^{\circ}$, results becomes quite different. A strong reflection drop is found near 1.9 , corresponding to the cut-off of the $F(1,2)$-like mode. 


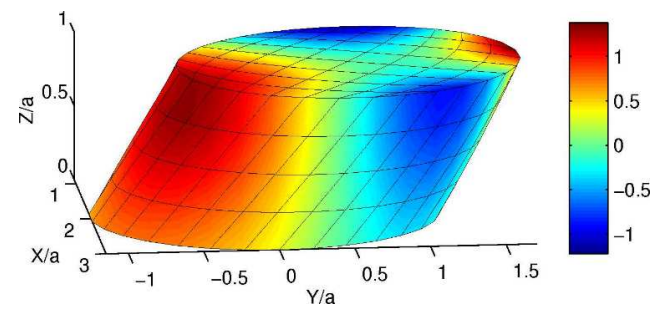

FIG. 6. Reflection of the first compressional-like mode by a free-end in a helical waveguide of angle $\phi=30^{\circ}$ : real part of the axial displacement at $\omega a / c_{s}=2$ (color online).

Figure 6 shows the color plot of the axial displacement $\left(u_{t}=u_{Z}\right)$ computed at $\omega a / c_{s}=2$. The global motion observed at this frequency is clearly not purely extensional, indicating significant conversions into non-compressional modes.

\section{E. Validation tests}

Though the power balance is well-satisfied in the previous simulations, this criterion is surely not sufficient to fully validate the proposed hybrid method and check the accuracy of results. In the following, two validation tests are performed.

\section{Infinite helical waveguide}

A first test-case consists in checking that a single incident mode travels without conversion or reflection in an infinite helical waveguide. The larger angle $\left(\phi=30^{\circ}\right)$ is considered. Figure 7 shows the hybrid FE-SAFE mesh. In that case, the transparent boundary $\Sigma$ is given by $\Sigma_{1} \cup \Sigma_{2}$, respectively corresponding to the bottom and top cross-sections. SAFE computations must be then performed on both $\Sigma_{1}$ and $\Sigma_{2}$. For clarity, $\mathbf{B}_{u}^{ \pm}$and $\mathbf{B}_{f}^{ \pm}$should now be understood as:

$$
\mathbf{B}_{u}^{ \pm}=\left[\begin{array}{cc}
{ }_{1} \mathbf{B}_{u}^{ \pm} & \mathbf{0} \\
\mathbf{0} & { }_{2} \mathbf{B}_{u}^{ \pm}
\end{array}\right] \quad \mathbf{B}_{f}^{ \pm}=\left[\begin{array}{cc}
{ }_{1} \mathbf{B}_{f}^{ \pm} & \mathbf{0} \\
\mathbf{0} & { }_{2} \mathbf{B}_{f}^{ \pm}
\end{array}\right]
$$

where ${ }_{i} \mathbf{B}_{u, f}^{ \pm}$denotes modal bases associated with $\Sigma_{i}$. 


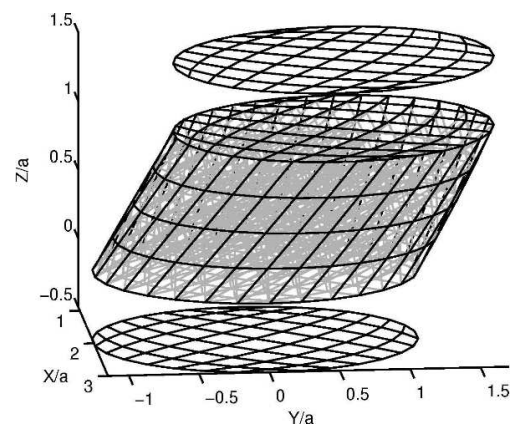

FIG. 7. Mesh of the infinite helical waveguide $\left(R=2 a, \phi=30^{\circ}\right)$. Bottom and top boundaries are both transparent.
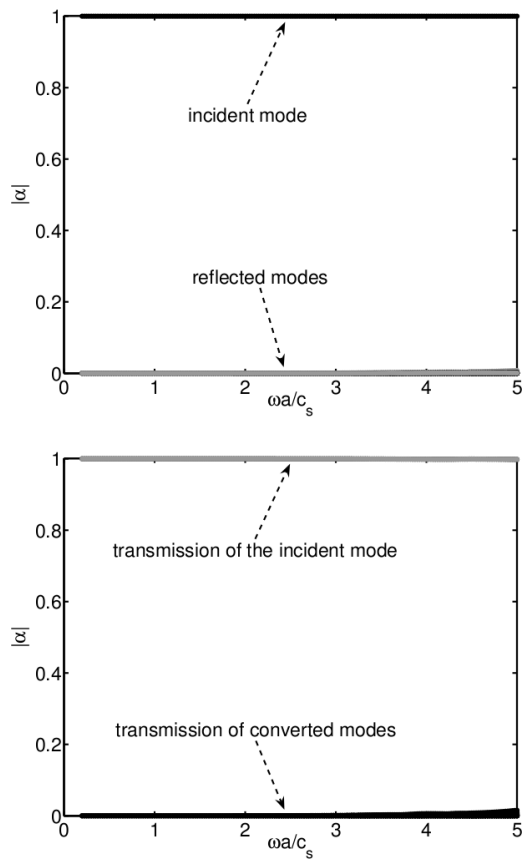

FIG. 8. Modal coefficient vs. frequency for $\phi=30^{\circ}$. Top: reflection, bottom: transmission.

Figure 8 gives the absolute value of the modal amplitudes of displacement, obtained in reflection (on $\Sigma_{1}$ ) and transmission (on $\Sigma_{2}$ ). The incident mode is still the $L(0,1)$-like mode, launched with a unit modal amplitude for all frequencies. As can be observed, this mode is almost fully transmitted, without reflection nor conversion, for the whole frequency range $[0 ; 5]$. Note that this test might also be viewed as a numerical way of checking that guided modes truly exist inside helical structures. 


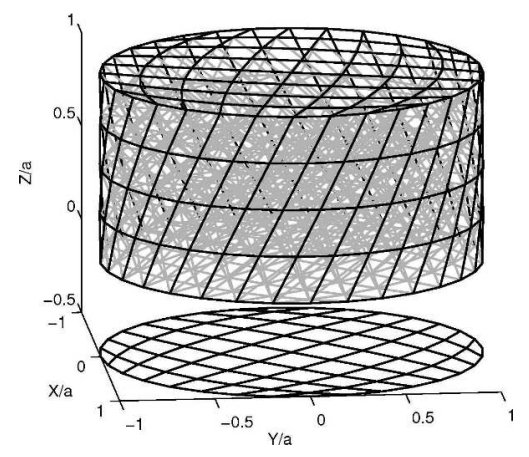

FIG. 9. Mesh used for the free-end twisted cylinder test.

It could be observed that the coefficients associated with reflected or transmitted converted modes tend to increase for highest frequencies. This is an expected result with FE techniques (the FE size should be diminished as the frequencies increases). The worst power balance error was $0.3 \%$ for that test.

\section{Free-end twisted cylinder}

The second validation test concerns the free-end cylinder, for which reference results are available in the literature ${ }^{54}$. This example has already been analyzed in Sec. IV.D, where guided modes were computed for a straight waveguides $(\kappa=\tau=0)$. Here, the main difference is that guided modes are computed with an arbitrary twisted coordinate system, which allows to fully validate the hybrid approach proposed in this paper for helical waveguides.

As explained in Sec. II.E, $\tau$ can be any value. We choose a high torsion value, $\tau a=0.5$. Figure 9 shows the FE mesh used for the test. In order to harden the test, the mesh of the FE portion is also twisted instead of being straight. The dispersion curves computed on $\Sigma$ are identical to Fig. 3 in the cylinder case and are not shown for conciseness.

Figure 10 exhibits the power reflection coefficients computed for the dimensionless frequency range [3.1;4.2], divided into 150 equal steps, with the $L(0,1)$ mode as incident. This frequency range focuses on the band where the $L(0,1)$ mode becomes converted to $L(0,2)$ 


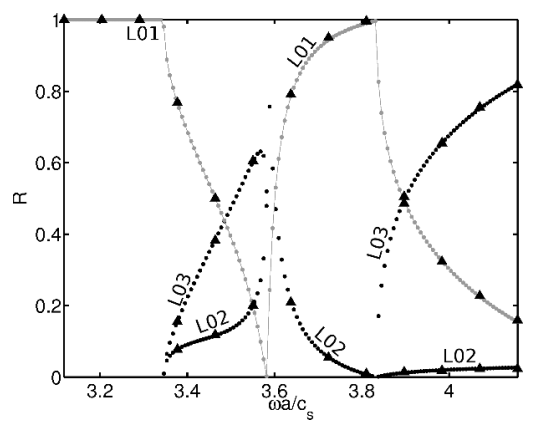

FIG. 10. Power reflection coefficient vs. frequency. The $L(0,1)$ mode is colored gray. Triangles: results of Gregory et al. [54].

and $L(0,3)$. For comparison, the results of Gregory et al. [54], obtained by means of analytical and least-square techniques, are superposed and all modes are explicitly labeled. Our numerical result are in quite good agreement, both qualitatively and quantitatively (note that the cylinder case in Fig. 5 also coincides with these results).

\section{CONCLUSION}

In this paper, the propagation of guided modes in curved waveguides and their scattering by inhomogeneities have been investigated. First, the existence of propagation modes traveling in curved waveguides has been highlighted in a general framework. Provided that the cross-section shape and medium properties do not vary along the waveguide axis, it has been proved that a sufficient condition for translational invariance is the independence on the axial coordinate of the metric tensor.

Then, the study has been focused on the elastodynamics of helical waveguides. Guided modes have been calculated thanks to a semi-analytical finite element method. For their scattering by inhomogeneities, a hybrid finite element method has been proposed. The technique is based on modal expansions at boundaries of the FE model, yielding transparent conditions. Some results have been shown for the scattering of the first extensional mode in free-end helical waveguides. Two validation tests have successfully been performed, demonstrating a good accuracy of the approach with acceptable computational cost. The present 
work paves the way for studies of wave-damage interaction in helical multi-wire strands.

Regardless waveguide curvature, three-dimensional numerical tools are becoming essential to study guided wave scattering in complex-shaped waveguides and defects. The proposed 3D hybrid FE-SAFE method, relatively simple to implement and computationally little expensive, offers an interesting technique for defect classification and sizing by guided waves.

\section{References}

1 D. Gridin, R. V. Craster, J. Fong, M. J. S. Lowe, and M. Beard, "The high-frequency asymptotic analysis of guided waves in a circular elastic annulus", Wave Motion 38, 67-90 (2003).

2 J. Qu, Y. Berthelot, and Z. Li, "Dispersion of guided circumferential waves in a circular annulus", in Review of Progress in Quantitative NDE, edited by D. O. Thompson and D. E. Chimenti, volume 15, 169-176 (Plenum Press, New York) (1998).

3 G. Liu and J. Qu, "Transient wave propagation in a circular annulus subjected to transient excitation on its outer surface", Journal of the Acoustical Society of America 104, 1210-1220 (1998).

4 A. Demma, P. Cawley, and M. Lowe, "The effect of bends on the propagation of guided waves in pipes", Journal of Pressure Vessel Technology 127, 328-335 (2005).

5 S. Finneveden and M. Fraggstedt, "Waveguide finite element for curved structures", Journal of Sound and Vibration 312, 644-671 (2008).

6 S. Towfighi and T. Kundu, "Elastic wave propagation in anisotropic spherical curved plates", International Journal of Solids and Structures 40, 5495-5510 (2003).

7 Y. Jiangong, W. Bin, H. Hongli, and H. Cunfu, "Characteristics of guided waves in anisotropic spherical curved plates", Wave Motion 44, 271-281 (2007).

8 A.-C. Hladky-Hennion, "Finite element analysis of the propagation of acoustic waves in waveguides", Journal of Sound and Vibration 194, 119-136 (1996). 
9 O. Onipede and S. B. Dong, "Propagating waves and end modes in pretwisted beams", Journal of Sound and Vibration 195, 313-330 (1996).

10 W. H. Wittrick, "On elastic wave propagation in helical springs", International Journal of Mechanical Sciences 8, 25-47 (1966).

11 J. Lee and D. J. Thompson, "Dynamic stiffness formulation, free vibration and wave motion of helical springs", Journal of Sound and Vibration 239, 297-320 (2001).

12 F. Treyssède, "Numerical investigation of elastic modes of propagation in helical waveguides", Journal of the Acoustical Society of America 121, 3398-3408 (2007).

13 F. Treyssède and L. Laguerre, "Investigation of elastic modes propagating in multi-wire helical waveguides", Journal of Sound and Vibration 329, 1702-1716 (2010).

14 W. Sollfrey, "Wave propagation on helical wires", Journal of Applied Physics 22, 905-910 (1951).

15 A. Nicolet, F. Zola, and S. Guenneau, "Modeling of twisted optical waveguides with edge elements", The European Physical Journal Applied Physics 28, 153-157 (2004).

16 A. Nicolet and F. Zola, "Finite element analysis of helicoidal waveguides", IET Science, Measurement and Technology 28, 67-70 (2007).

17 H. Kwun, K. A. Bartels, and J. J. Hanley, "Effects of tensile loading on the properties of elastic-wave propagation in a strand", Journal of the Acoustical Society of America 103, 3370-3375 (1998).

18 L. Laguerre, M. Brissaud, and J.-C. Aime, "Magnetostrictive pulse-echo device for nondestructive evaluation of steel cylindrical materials using guide waves", Ultrasonics 39, $503-514(2002)$.

19 M. D. Beard, M. J. S. Lowe, and P. Cawley, "Ultrasonic guided waves for inspection of grouted tendons and bolts", Journal of Materials in Civil Engineering 212, 212-218 (2003).

20 P. Rizzo and F. L. di Scalea, "Load measurement and health monitoring in cable stays via guided wave magnetostrictive ultrasonics", Materials Evaluation 62, 1057-1065 (2004).

21 "Guided ultrasonic waves for non-destructive monitoring of the stress levels in prestressed 
steel strands", Ultrasonics 49, 162-171 (2009).

22 L. Gavric, "Computation of propagative waves in free rail using a finite element technique", Journal of Sound and Vibration 185, 531-543 (1995).

23 T. Hayashi, W.-J. Song, and J. L. Rose, "Guided wave dispersion curves for a bar with an arbitrary cross-section, a rod and rail example", Ultrasonics 41, 175-183 (2003).

24 V. Damljanovic and R. L. Weaver, "Propagating and evanescent elastic waves in cylindrical waveguides of arbitrary cross-section", Journal of the Acoustical Society of America 115, 1572-1581 (2004).

25 I. Bartoli, A. Marzani, F. L. di Scalea, and E. Viola, "Modeling wave propagation in damped waveguides of arbitrary cross-section", Journal of Sound and Vibration 295, 685-707 (2006)

26 P. E. Lagasse, "Higher-order finite element analysis of topographic guides supporting elastic surface waves", Journal of the Acoustical Society of America 53, 1116-1122 (1973).

27 S. K. Datta, A. H. Shah, R. L. Bratton, and T. Chakraborty, "Wave propagation in laminated composite plates", Journal of the Acoustical Society of America 83, 2020$2026(1988)$.

28 R. J. Astley and A. Cummings, "A finite element scheme for attenuation in ducts lined with porous material: comparison with experiment", Journal of Sound and Vibration 116, 239-263 (1987).

29 R. Kirby, "Transmission loss predictions for dissipative silencers of arbitrary cross-section in the presence of mean flow", Journal of the Acoustical Society of America 114, 200-209 (2003).

30 S. Guenneau, A. Nicolet, F. Zolla, and S. Lasquellec, "Modeling of photonic crystal optical fibers with finite elements", IEEE Transactions on Magnetics 38, 1261-1264 (2002).

31 F. Treyssède, "Elastic waves in helical waveguides", Wave Motion 121, 457-470 (2008).

32 Y. Al-Nassar, S. Datta, and A. Shah, "Scattering of lamb waves by a normal rectangular strip weldment", Ultrasonics 29, 125-132 (1991).

33 W. Karunasena, A. Shah, and S. Datta, "Plane-strain-wave scattering by cracks in lam- 
inated composite plates", Journal of Engineering Mechanics 117, 1738-1754 (1991).

34 A. Mal and Z. Chang, "A semi-numerical method for elastic wave scattering calculations", Geophysical Journal International 143, 328-334 (2000).

35 W. Karunasena, K. Liew, and S. Kitipornchai, "Hybrid analysis of lamb wave reflection by a crack at the fixed edge of a composite plate", Computer Methods in Applied Mechanics and Engineering 125, 221-233 (1995).

36 J. M. Galan and R. Abascal, "Lamb mode conversion at edges. a hybrid boundary element-finite element solution", Journal of the Acoustical Society of America 117, 1777$-1784(2005)$.

37 Y. Cho and J. L. Rose, "A boundary element solution for a mode conversion study on the edge reflection of lamb waves", Journal of the Acoustical Society of America 99, 2097-2109 (1996).

38 N. Rattanawangcharoen, W. Zhuang, A. Shah, and S. Datta, "Axisymmetric guided waves in jointed laminated cylinders", Journal of Engineering Mechanics 123, 1020-1026 (1997).

39 R. Kirby, "Modeling sound propagation in acoustic waveguides using a hybrid numerical method", Journal of the Acoustical Society of America 124, 1930-1940 (2008).

40 R. J. Astley, "Fe mode-matching schemes for the exterior helmholtz problem and their relationship to the fe-dtn approach", Communications in Numerical Methods in Engineering 12, 257-267 (1996).

41 M. J. S. Lowe, D. N. Alleyne, and P. Cawley, "Defect detection in pipes using guided waves", Ultrasonics 36, 147-154 (1998).

42 J. Ma, F. Simonetti, and M. Lowe, "Scattering of the fundamental torsional mode by an axisymmetric layer inside a pipe", Journal of the Acoustical Society of America 120, 1871-1880 (2006).

43 L. Moreau, M. Castaings, B. Hosten, and M. Predoi, "An orthogonality relation-based technique for post-processing finite element predictions of waves scattering in solid waveguides", Journal of the Acoustical Society of America 120, 611-620 (2006). 
44 M. Predoi, M. Castaings, and L. Moreau, "Influence of material viscoelasticity on the scattering of guided waves by defects", Journal of the Acoustical Society of America 124, 2883-2894 (2008).

45 D. Chapelle and K. J. Bathe, The Finite Element Analysis of Shells - Fundamentals (Springer-Verlag, Berlin) (2003), 330 pp.

46 A. Gray, E. Abbena, and S. Salamon, Modern Differential Geometry of Curves and Surfaces with Mathematica, 3rd edition (Chapman \& Hall, Boca Raton) (2006), 984 pp.

47 F. Tisseur and K. Meerbergen, "The quadratic eigenvalue problem", SIAM Review 43, $235-286$ (2001).

48 B. Auld, Acoustic Fields and Waves in Solids, volume I, 2nd edition (Krieger, Malabar) (1990), 446 pp.

49 J. D. Achenbach, Wave Propagation in Elastic Solids (North-Holland, Amsterdam) (1973), 425 pp.

50 A. Bernard, M. J. S. Lowe, and M. Deschamps, "Guided waves energy velocity in absorbing and non-absorbing plates", Journal of the Acoustical Society of America 110, 186-196 (2001).

51 A. H. Meitzler, "Mode coupling occuring in the propagation of elastic pulses in wires", Journal of the Acoustical Society of America 33, 435-445 (1961).

52 A. Gunawan and S. Hirose, "Mode-exciting method for lamb wave-scattering analysis", Journal of the Acoustical Society of America 115, 996-1005 (2004).

53 B. Auld, Acoustic Fields and Waves in Solids, volume II, 2nd edition (Krieger, Malabar) (1990), $432 \mathrm{pp}$.

54 R. Gregory and I. Gladwell, "Axisymmetric waves in a semi-infinite elastic rod", Quaterly Journal of Mechanics and Applied Mathematics 42, 327-337 (1989). 


\section{List of Figures}

FIG. 1 Helix centerline curve (one step) with its associated Serret-Frenet basis and $(x, y, s)$ helical coordinate system. . . . . . . . . . . . 9

FIG. $2 Z=0$ plane-cut of a helical waveguide $R=2 a$ having a circular cross-section of radius $a$ for the following lay angles: $\phi=0^{\circ}$ (cylinder), $15^{\circ}$ and $30^{\circ}$ (from left to right). The SAFE mesh used for the computations is also shown. . . 11

FIG. 3 From left to right: dimensionless energy velocity vs. frequency for $\phi=0^{\circ}$ (cylinder), $15^{\circ}$ and $30^{\circ}$. The first compressional-like mode is colored gray. . .

FIG. 4 Geometry and FE mesh used for the computations with the hybrid method for a free-end helical waveguide with $R=2 a$ and the following lay angles: $\phi=0^{\circ}$ (cylinder), $15^{\circ}$ and $30^{\circ}$ (from left to right). The bottom cross-section, here duplicated in the plane $Z=-0.5$, corresponds to $\Sigma$ (transparent condition). The remaining boundaries of the FE model are free, including the top crosssection representing the free-end. . . . . . . . . . . .

FIG. 5 From left to right: power reflection coefficient vs. frequency for $\phi=0^{\circ}$ (cylinder), $15^{\circ}$ and $30^{\circ}$. The first compressional-like mode is colored gray. . . . . .

FIG. 6 Reflection of the first compressional-like mode by a free-end in a helical waveguide of angle $\phi=30^{\circ}$ : real part of the axial displacement at $\omega a / c_{s}=2$ (color online). . . . . . . . . . . . . . . . . .

FIG. 7 Mesh of the infinite helical waveguide $\left(R=2 a, \phi=30^{\circ}\right)$. Bottom and top boundaries are both transparent.

FIG. 8 Modal coefficient vs. frequency for $\phi=30^{\circ}$. Top: reflection, bottom: transmission.

FIG. 9 Mesh used for the free-end twisted cylinder test. . . . . . . . . . . . . . . . 26

FIG. 10 Power reflection coefficient vs. frequency. The $L(0,1)$ mode is colored gray. Triangles: results of Gregory et al. [54]. . . . . . . . . . . . . . . . . . . 